\title{
MISE EN SCÈNE DU MONDE POLITIQUE DANS LES ÉMISSIONS TÉLÉVISÉES ITALIENNES
}

\begin{abstract}
Andrea Caretta et Valeria Pangrazio ${ }^{1}$
Dans la perspective de cette étude, le cas italien est intéressant parce qu'il dévoile les enjeux du rapport entre la politique et la télévision en démontrant comment la communication politique change à cause de l'emploi toujours plus massif de la télévision. L'entrée en 1994 de Silvio Berlusconi - propriétaire de chaînes de télévision privées, mais aussi de journaux et de maison d'édition - dans la vie politique italienne, a augmenté l'intérêt des hommes politiques pour les mass media, en particulier la télévision. Cet article vise à donner des éléments de compréhension sur l'état de la communication politique italienne à la télévision et de ses liens avec les émissions de divertissement. Dans un premier temps, une analyse quantitative basée sur des données de l'Osservatorio di Pavia ${ }^{2}$ dépeindra l'évolution de la politique à la télévision ces dernières années. Ensuite, nous présenterons

1 Osservatorio di Pavia

2 L'Osservatorio di Pavia, institut de recherche et d'analyse de la communication, travaille dans le domaine de la recherche sur le pluralisme social, culturel et politique dans les moyens de communication, en collaboration avec l'Università degli Studi de Pavie. Par ailleurs il a établi un long rapport de collaboration avec la télévision publique. L'Osservatorio est chargé par la RAI du monitoring sur le pluralisme politique à la télévision. Les données obtenues sont utilisées par la Commission Parlementaire de Vigilance sur la RAI. Pour en savoir en plus : http:// www.osservatorio.it.
\end{abstract}

Recherches en communication, $\mathrm{n}^{\circ} 24$ (2005). 
une étude de cas sur le dernier événement médiatique en date en Italie : l'émission de divertissement Rockpolitick. Nous reviendrons également sur ses conséquences médiatiques, notamment par rapport au principal programme d'information politique italien, Porta a porta.

\section{Quelques données quantitatives}

Avant tout, il nous faut expliquer les variables qui sont utilisées par l'Osservatorio di Pavia dans son activité de monitoring de la chaîne publique italienne. Elles sont de deux ordres : le T et le GDT. Le T, ou temps d'attention, est mesuré en minutes et en secondes. Il s'agit de la durée pendant laquelle le Sujet Politique ${ }^{1}$ est le contenu du discours, que ce soit le sien ou celui d'autrui. Le GDT, lui aussi mesuré en minutes et secondes, est le temps pendant lequel le Sujet Politique parle directement au public. Autrement dit, le GDT mesure le temps où le Sujet Politique est présent en télévision. Les deux variables sont considérées comme des indicateurs du temps consacré à la communication politique à la télévision. Notre analyse porte sur quatre années, de 2000 à 2004.

Dans le Tableau 1.1 et dans le Graphique A, nous pouvons voir l'évolution des deux variables, le T et GDT, sur ce laps de temps par rapport au total de la programmation télévisée, 24 heures sur 24, 365 jours par an. Nous pouvons remarquer que, sur les chaînes publiques, la communication politique, directe et indirecte, a connu une évolution croissante.

Tableau 1.1 Evolution de la Communication Politique Total de la Communication Politique

\begin{tabular}{|r|c|c|}
\hline Année & T & GDT \\
\hline 2000 & 40.704 & 24.049 \\
\hline 2001 & 46.988 & 27.426 \\
\hline 2002 & 46.097 & 25.757 \\
\hline 2003 & 45.118 & 26.701 \\
\hline 2004 & 50.277 & 27.707
\end{tabular}

1 Est considéré comme Sujet Politique toute personne qui occupe une charge élective à l'intérieur des administrations publiques aux différents niveaux nationaux et locaux, ou tout qui occupe des charges de direction dans des partis politiques représentés dans le Parlemente ou dans les administrations publiques aux différents niveaux nationaux et locaux. 


\section{Évolution du Total de la communication politique (en minutes)}

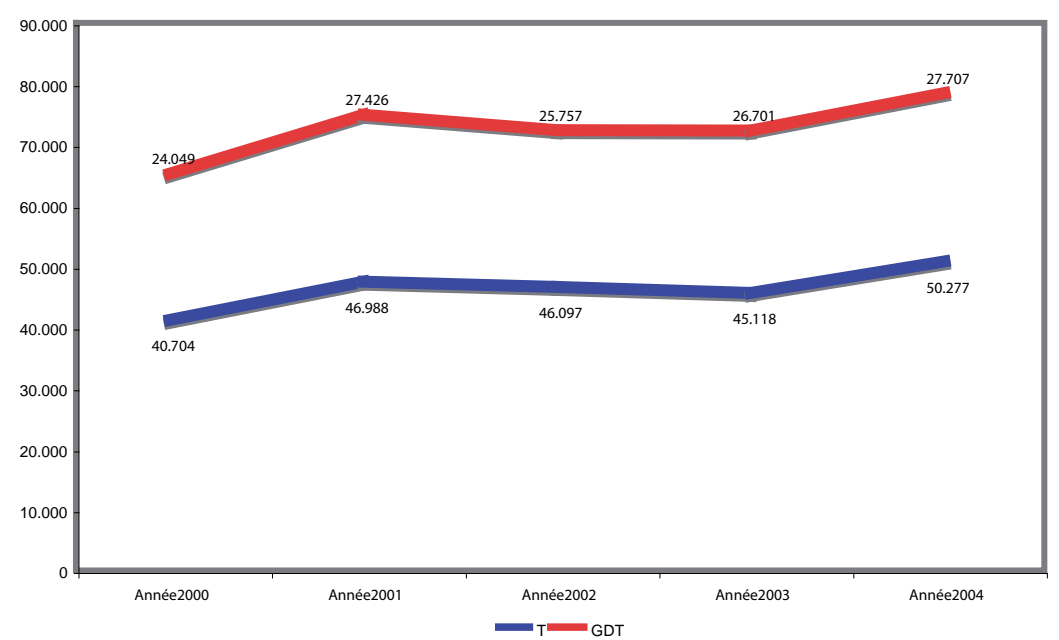

Ces tableaux englobent donc la totalité de la programmation télévisée. Ce qui nous intéresse dans le contexte de cette étude, ce sont les émissions dédiées à l'information politique, que nous nommerons « information», et celles de divertissement où les politiciens sont invités. Nous les indiquerons sous l'étiquette « autre». En examinant l'évolution de la présence des hommes politiques au sein de ces deux genres, nous pouvons répondre à la question initiale de notre étude « $\mathrm{La}$ communication politique se transforme-t-elle en spectacle? $\gg$ ou, pour rester dans le domaine de l'analyse quantitative, «La communication politique se déplace-t-elle vers les programmes liés au spectacle ?»

Le Tableau 1.2 indique une évolution très intéressante de la représentation du politique dans les émissions «autres ». Jusqu'en 2002, la croissance est soutenue pour ensuite baisser en 2003 et 2004. Cette remarque vaut à la fois pour le T et le GDT.

Tableau 1.2 Evolution de la Communication Politique dans Autre

\begin{tabular}{|r|c|c|}
\hline Année & $\mathrm{T}$ & GDT \\
\hline 2000 & $16 \%$ & $17 \%$ \\
\hline 2001 & $25 \%$ & $26 \%$ \\
\hline 2002 & $28 \%$ & $31 \%$ \\
\hline 2003 & $24 \%$ & $24 \%$ \\
\hline 2004 & $16 \%$ & $16 \%$
\end{tabular}

* données en pourcentage sur le total de la communication politique 
Nous devons ensuite aborder la question de l'apparition de personnalités politiques au sein d'émissions qui dépassent le cadre politique au sens strict, et nous tenterons de voir si cette démarche est un phénomène typiquement italien. Le Tableau 1.3 montre l'évolution de la communication politique dans les transmissions consacrées à l'Information.

Tableau 1.3 Evolution de la Communication Politique dans Information

\begin{tabular}{|r|c|c|}
\hline Année & $\mathrm{T}$ & GDT \\
\hline 2000 & $16 \%$ & $20 \%$ \\
\hline 2001 & $15 \%$ & $19 \%$ \\
\hline 2002 & $17 \%$ & $23 \%$ \\
\hline 2003 & $18 \%$ & $26 \%$ \\
\hline 2004 & $22 \%$ & $32 \%$ \\
\hline
\end{tabular}

* données en pourcentage sur le total de la communication politique

L'évolution est contraire à celle décrite dans le tableau relatif au genre «autre». L'accroissement de la communication politique, enregistré en 2003 et en 2004 au sein d'émissions dédiées au cadre politique au sens strict, semble signaler une "migration" des politiciens vers des espaces institutionnellement consacrés au politique dans les grilles de programmes. Si nous nous arrêtons à ces données, nous pouvons conclure que les politiciens ont tendance à réinvestir les émissions politiques, contrairement à ce qu'on a pu observer il y a quelques années. Les politiciens désertent les émissions où l'on montrait leur côté humain, "amical", plus proche des citoyens, où l'on discutait de thèmes "frivoles", semblant souligner que cela n'est pas lié à l'activité normale des représentants publics. Ils reviennent maintenant se confronter aux journalistes, aux experts et à leurs collègues pour débattre de "thèmes sérieux".

Mais est-ce vraiment le cas, ou bien ces données cachent-elles une transformation plus structurelle de la communication politique ? Pour le découvrir, nous devons examiner les thèmes qui sont traités dans les émissions que nous avons classées comme dans la catégorie « information ». Nous le ferons à travers l'étude d'un cas : Porta a porta.

\section{Les thèmes de l'information}

Porta a porta est en fait le lieu télévise privilégié du débat politique en Italie. L'émission a été créée en 1996 et est animée par le journaliste Bruno Vespa. Le programme est défini comme « le bon salon de 
la politique italienne », dans sa volonté de montrer le débat politique en suivant les schémas institutionnels.

Cette émission très populaire est considérée par les leaders politiques comme un lieu privilégié où manifester leurs points de vue et ceux de leur parti. Elle est donc une vitrine pour les personnages politiques italiens. Mais Porta a porta se caractérise également comme un véritable lieu politique. On ne fait pas qu'y analyser la vie politique, elle s'y produit. Des événements politiques, des déclarations, des prises de position inattendues se déroulent parfois sur le plateau. Les journalistes, un peu polémiques, vont jusqu'à qualifier le programme de « troisième chambre de la politique italienne ».

Nous avons donc examiné les thèmes discutés par les politiciens invités dans l'émission Porta a porta de 2003 à 2005. Ils relèvent de deux catégories. Sous l'étiquette "politique», nous avons classé les sujets qui appartiennent à l'activité politique et du gouvernement. L'économie, la justice, les alliances entre les partis, la politique étrangère notamment y sont rassemblées. La deuxième rubrique, que nous appellerons «autre», reprend tous les faits divers, les événements people, la vie personnelle des hommes et femmes politiques. Les résultats sont synthétisés dans le Tableau 1.4 et le Graphique 4. Au fil des années, les invités de Bruno Vespa ont de plus en plus parlé des thèmes qui ne font pas partie du domaine politique au sens strict.

\begin{tabular}{l|rrr} 
Tableau 1.4 & Porta a Porta: Théme de la Communication Politique \\
Année & 2003 & 2004 & 2005 \\
\hline Théme & $\%$ & $\%$ & $\%$ \\
\hline Autres & $26,33 \%$ & $29,23 \%$ & $35,98 \%$ \\
\hline Politiques & $73,67 \%$ & $70,77 \%$ & $64,02 \%$ \\
\hline Total & $100,00 \%$ & $100,00 \%$ & $100,00 \%$
\end{tabular}

* données en pourcentage sur le total de la communication politique dans Porta a Porta

En conclusion, nous pouvons affirmer que le GDT, donc la présence des politiciens en télévision, se concentre de plus en plus dans les programmes dédiés à l'information politique (on passe de $19 \%$ en 2001 à 32\% en 2004) alors que la présence globale des politiciens à la télévision reste constante (voir le Tableau 1.1). Cependant, les éléments repérés dans le Tableau 1.3 relativisent ces affirmations : les thèmes abordés ne relèvent pas toujours du domaine strictement politique. 


\section{Thèmes Autres et Politiques dans Porta a Porta}

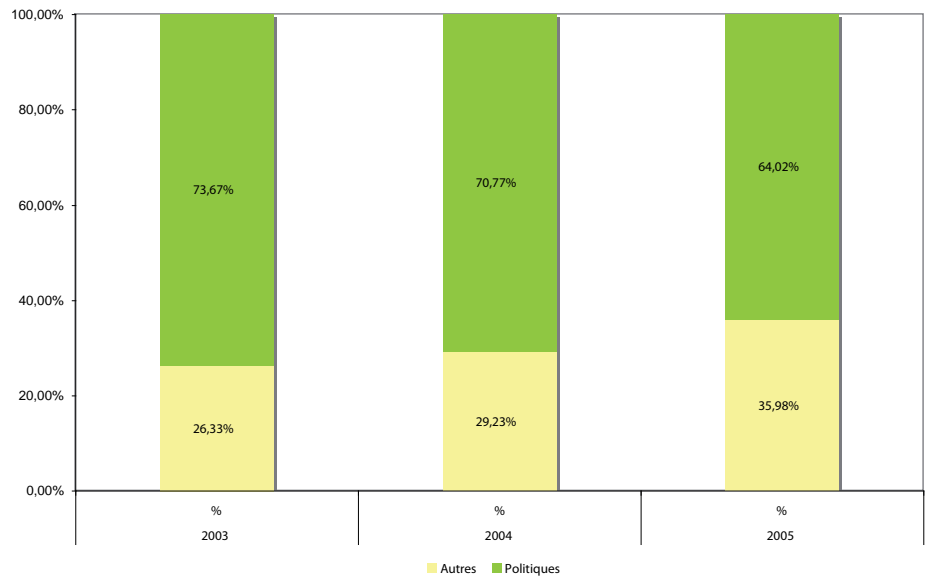

\section{Quelques conclusions}

Avant de tirer quelques conclusions, un détour par le contexte de l'information à la télévision italienne est nécessaire. Ces dernières années, la manière dont on fait de la communication politique, plus précisément encore la manière dont les politiciens se présentent en télévision, a changé. Et cela semble avoir eu des conséquences sur le genre « émission d'information » où le politique est normalement cantonné.

Ces dernières années, le genre «information » a évolué vers de plus en plus de spectacularisation. Cela s'est surtout ressenti dans la façon dont les politiciens ont été représentés, dans leur "mise en scène". Jusque dans les années nonante, dans la période de la Première République, le rôle du journaliste était ancillaire et son attitude restait respectueuse. Après l'enquête «mani pulite »(1992) qui a marqué la fin de la Première République, les rapports entre les journalistes et les politiciens sont devenus beaucoup plus agressifs. Une certaine pression s'est exercée sur les politiciens qui ont été véritablement "interrogés". Le public était encouragé à exprimer son accord, mais aussi son désaccord vis à vis des déclarations des invités.

Entre 1996 et 2001, les relations entre journalistes et politiciens se sont à nouveau apaisées. Les hommes et femmes politiques sont maintenant présentés en tant que représentants du pouvoir ou des institutions, mais ils sont aussi "mis en scène" au moyen leur vie personnelle, leur côté humain, leurs passions. On montre l'homme qui se cache derrière le politicien. Cette recherche d' "humanité" est principalement due à 
deux facteurs. Tout d'abord, le changement du système électoral passé au système ; ensuite, la victoire de Silvio Berlusconi aux élections de 1994 remportées grâce à une campagne axée sur l'"homme" et sur le fait qu'il ne provenait pas du milieu politique.

Née en 1996, Porta a porta illustre de manière exemplaire cette transformation de la communication politique. Durant les années « héroïques », les téléspectateurs ont pu comtempler Umberto Bossi (leader de la Lega Lombarda) cuisiner la polenta, Massimo D’Alema (leader des Démocrates de gauche) concocter un risotto et Giuliano Amato (alors chef du gouvernement) faire du tennis sur le plateau de Bruno Vespa. Dans ces années du "triomphe de l'humanité", on pourrait également pointer la célèbre cérémonie de la signature du « contrat avec les Italiens» ${ }^{1}$, signé par Berlusconi en 2001. Un bel exemple de communication politique où la confiance dans la personne et ses capacités devient la base du rapport entre électeurs et élus.

Dès les années nonante, la communication politique en télévision a intégré à ses codes ceux du monde du spectacle. Au début, la scénographie et les scénarii des émissions ont été améliorés par des éléments puisés au monde du théâtre où le politicien était "mis en scène" devant un public qui le jugeait. Puis, à partir de la moitié de la décennie, la "mise en scène" du politique s'est déplacée du théâtre vers l'amusement. Le politicien va alors s'exhiber dans des lieux télévisés inhabituels pour lui. Cependant, les émissions d'information, malgré une forme plus influencée par le monde du spectacle, véhiculaient toujours un contenu politique. Nous voyons actuellement que l'évolution de la communication politique, cela est confirmé par les données, montre que la nature même du genre « information » est en train de se modifier. Le genre « information » ne se définit plus comme un esemble d'émissions où politiciens et experts s'affrontent sur des thèmes politiques, mais il devient un ensemble d'émission où les politiciens sont présents, indépendamment des sujets abordés, politiques ou non. La dérive autoréférentielle des émissions d'information - devenues le lieu, non plus de la politique, mais des politiciens - laisse un vide dans la programmation télévisée. Finalement où aborde-t-on les thèmes politiques ? Ce vide semble pouvoir être comblé (est-ce un bien, est-ce un danger ?) par les

1 Quelques jours avant les élections législatives du 2001, Berlusconi signe en direct dans Porta a porta un contrat avec les Italiens dans lequel il s'engage, en cas de victoire, à réaliser les 5 promesses faites pendant la campagne électorale. 
animateurs du divertissement qui construisent leurs shows autour des personnalités de leurs invités.

\section{Rockpolitica et Porta a porta : la fin d'un genre télévisé}

Rockpolitick est une émission qui se situe entre la variété et la satire. Le programme, animé par le présentateur vedette Adriano Celentano, a créé l'événement médiatique en 2005. Il a entretenu le débat dans les journaux et à la une des journaux télévisés. Les réactions ont été diverses. La droite le critique pour ses attaques contre Silvio Berlusconi. La gauche le loue pour sa liberté de ton. Quelques éléments d'explication sur le contexte politique et médiatique italien sont nécessaires pour comprendre cet engouement. En effet, le David Letterman Show aux Etats-Unis, par exemple, parle de la politique et en parle violemment sans qu'il soit devenu le centre des débats comme c'est le cas pour Rockpolitick. Une brève description des termes intervenant dans l'émission et dans ses échos (mais aussi dans le langage politique courant en Italie) sera utile pour comprendre la dynamique qui a généré le cas Rockpolitick.

Rockpolitick d'une part illustre et de l'autre met en scène l'anomalie italienne, la spécificité de sa situation politico-médiatique ${ }^{1} \mathrm{du}$ pays. En Italie, on peut parler d'une intervention excessive du gouvernement dans la gestion de la télévision publique et d'une véritable confusion créée par la collusion entre média et politique ${ }^{2}$.

On retrouve d'une part la logique de la « lottizzazione », c'est-àdire la séparation des chaînes publiques et de leurs postes de responsabilité déterminée par les partis politiques, notamment par la coalition au gouvernement. Cela a conduit à une constante intervention de la politique sur la télévision publique et à une domination régie par la loi de l'information par les partis politiques. D'un autre côté, le pouvoir s'est

1 Pour une analyse détaillée, voir la partie sur l'Italie, rédigée par Gianpiero Mazzoleni, recherche de l' Open Society Institut « Television across Europe, regulation, policy and indipendence» : http://www.eumap.org/topics/media/television_europe/ national/italy/media_ita2.pdf

2 En 2004 deux résolutions, une du Parlement européen (résolution du 24 avril 2004) et l'autre du Conseil de l'Europe (résolution du 3 juin 2004), ont signalé l'anomalie italienne, en mettant en évidence le déficit d'indépendance du service public et en manifestant des préoccupations à propos de l'état du pluralisme en Italie. 
exceptionnellement concentré. La famille de Silvio Berlusconi possède les chaînes privées nationales (Canale 5, Rete 4, Italia 1). D'autant que, selon la loi italienne sur la télévision publique, le chef du gouvernement, contrôle indirectement les chaînes de la télévision publique. Le régime du duopôle entre la télévision publique et la télévision privée de Berlusconi, grâce auquel la RAI et MEDIASET se partagent la majorité des ressources publicitaires et de l'audience, rend cette situation plus délicate.

Ceci explique l'importance que les moyens d'information ont pris dans la politique italienne. Le thème de la télévision est central dans les discussions politiques en Italie et notamment le problème du conflit d'intérêt qu'incarne Silvio Berlusconi (il est à la fois un entrepreneur dans le secteur des médias et le chef du gouvernement). Le débat s'est intensifié quand le gouvernement de centre gauche (19962001) a échoué à apporter une solution au problème et que les critiques se sont focalisés sur la solution envisagée par le gouvernement de droite (actuellement au pouvoir, et dont Silvio Berlusconi est le chef).

\section{L'événement médiatique}

Rockpolitick a été diffusée de 21 heures à minuit quatre jeudi consécutifs en octobre et novembre 2005 (précisément les 20 et 27 octobre, et les 3 et 10 novembre) sur la RAI 1, la chaîne principale de la télévision publique. Le programme a remporté un gros succès d'audience. En moyenne, onze millions de téléspectateurs ont regardé les quatre émissions (mais le programme à connu des pics qui sont montés jusqu'à 15 millions de téléspectateurs). Avant sa diffusion, les journaux, les personnalités du monde du spectacle et de la télévision, les institutions ont largement parlé de l'émission, ce qui a excité la curiosité de l'opinion publique. S'il était très attendu, le programme était également l'objet de polémiques. À tel point que le directeur de la chaîne s'est "auto-suspendu" de ses fonctions pour la durée de l'émission. À moins de six mois des élections législatives où s'affronteront Silvio Berlusconi et Romano Prodi, le programme a fait l'effet d'une petite bombe.

Le titre est déjà indicateur du genre de produit télévisé offert. L'association du rock et de la politique classe le programme dans le divertissement. Rockpolitick: le titre suggère une nouvelle façon de produire du spectacle et de parler de politique. 
Le succès de l'émission est dû en partie à la présence d'invités de premier rang (des chanteurs comme Patti Smith, Carlos Santana, Eros Ramazzotti, Riccardo Cocciante ; des acteurs comme Gérard Depardieu et Roberto Benigni; des personnalités du monde du sport comme Valentino Rossi), mais surtout à son animateur: Adriano Celentano. C'est une personnalité présente sur la scène artistique italienne depuis les années 60 . Il est célèbre notamment pour sa carrière de chanteur, mais aussi en tant que présentateur. Ces dernières années, Celentano a présenté (mais aussi créé et produit) des émissions de divertissement de prime time qui ont connu un grand succès. Celentano a un style particulier, au départ peu approprié à la télévision pourtant. Il ponctue ses émissions de discours lents et longs qui semblent autant de pauses qui cassent le rythme du programme. Durant ces discours, il commente l'actualité, critique les valeurs de la société italienne, de la politique (d'où son surnom: "le prédicateur »). Celentano est souvent taxé de qualunquista ${ }^{1}$ et de populiste à cause son langage simple et de sa capacité d'incarner les humeurs des Italiens.

\section{Les quatre émissions et leurs thèmes}

Rockpolitick est une émission de divertissement qui alterne musique, ballet, invités célèbres du monde du spectacle, mais avec une caractéristique tout à fait particulière : elle parle de politique et en parle avec des langages différents.

Un premier niveau que l'on perçoit est le satirique. Celentano ne fait pas de la satire, mais l'émission est caractérisée par la présence des comiques qui épinglent dans leurs spectacles les politiciens et les institutions politiques. Nous ne nous attarderons pas sur cette dimension. La satire ne peut pas être analysée puisque sa fonction est uniquement de fournir du divertissement. À titre d'information, nous soulignons que sur environ 12 heures de diffusion pour les quatre premières émissions, les comiques ont parlé des personnalités de la politique durant 35 minutes. Ils se sont principalement focalisés sur Silvio Berlusconi, puisque 24 minutes lui ont été consacrées.

Un autre élément intéressant est l'utilisation des images. L'émission diffuse des images, soit liées à la politique en tant que telle (moments

1 Qualunquismo : attitude d'indifférence et de manque de confiance dans la politique et dans le système des partis, qui se manifeste par des prises de position simplistes envers l'Etat ou le gouvernement. 
clés de la politique italienne récente), soit qui réfèrent à la politique au sens large (des images liées aux thèmes comme la guerre ou l'environnement, par exemple). Certains mots apparaissent au sein des plans. Ils sont porteurs d'un sens symbolique. Ces images sont toujours accompagnées par l'orchestre présent sur le plateau - la musique joue souvent sur le contraste (par exemple, une musique gaie accompagne des images crues) - ou des ballets.

Il est intéressant de se pencher sur les thèmes évoqués durant les discours, même s'il n'est pas toujours facile d'en faire une description ordonnée et logique. En effet, dans ses longs discours, Celentano aborde - avec un langage opaque - des enjeux importants de la société italienne : les finances, les affaires, l'écologie et les abus dans le secteur du bâtiment. Il parle également des thèmes sociétaux plus larges comme les changements climatiques et les récentes catastrophes qu'ils ont occasionnées, les guerres (du Vietnam à l'invasion du Tibet), l'utilisation de l'arme nucléaire, la pauvreté dans le monde, etc. Le présentateur lance également quelques réflexions sur le rôle idéal de la politique et sur la démocratie. Tout ceci dans la perspective, souvent rappelée, des élections législatives d'avril 2006 et des municipales de Milan (par rapport auxquelles il donne son opinion).

Le moment le plus intense de Rockpolitick s'est déroulé lors de la première émission, quand le présentateur a parlé de la liberté d'expression. Nous l'avons déjà souligné, dans le contexte italien, les questions de la liberté d'expression et du pluralisme de l'information sont des sujets chauds de la politique. Ils sont devenus de vrais enjeux politiques, chers autant aux opposants de Berlusconi qui utilisent l'argument contre le Premier Ministre qu'à ceux qui le soutiennent et qui défendent la légimitié de la situation. Rockpolitick est entré dans cette discussion en créant un événement autour du retour à l'écran du journaliste Michele Santoro qui avait été exclu de la télévision publique parce qu'il avait critiqué le chef du gouvernement. Celentano a d'abord rediffusé la conférence de presse du 18 avril 2002, le célèbre "édit bulgare". Lors d'une visite en Bulgarie, Berlusconi a stigmatisé l'usage «criminel, factieux » du petit écran par trois personnalités de la télévision publique : Enzo Biagi (doyen modéré du journalisme télévisé), Daniele Luttazzi (auteur satirique) et Michele Santoro (journaliste d'investigation et présentateur d'émission à succès depuis les années nonante). À la rentrée, les trois personnalités visées avaient disparu de l'écran. Après cette rediffusion, le présentateur a proposé un classement rédigé 
par « Freedom house $»^{1}$ sur la liberté de presse dans le monde. Selon ce classement, l'Italie se trouve en 77ème position, entre la Bulgarie et la Mongolie, et est considérée comme un pays qui ne respecte que partiellement la liberté de la presse. Enfin, Michele Santoro a fait son entrée sur le plateau. Il n'a pas prononcé un discours explicitement politique, mais sa présence avait une valeur symbolique importante. Il faut souligner que Santoro a démissionné de son siège au Parlement européen la vieille de l'émission (une autre motivation pour les médias de parler de l'émission).

\section{Porta a porta regarde Rockpolitick}

Porta a porta est l'émission diffusée juste après Rockpolitick (du moins pour les deuxième, troisième et quatrième numéros, ce n'était pas le cas pour le premier). Le programme se structure autour de plusieurs invités. Porta a porta se structure habituellement autour de la présence des invités. Dans les trois émissions analysées, la présence du monde politique a été assez significative. Douze politiciens ont participé, autant de la majorité que de l'opposition. Parmi eux, Mario Landolfi, le ministre des Communications et Paolo Gentiloni, le président de la Commission parlementaire de contrôle de la télévision publique. Des personnalités du journalisme, du monde du spectacle et des experts des médias et de la communication étaient également invités. Durant l'émission, les participants doivent souvent prendre position sur un thème. Durant les trois émissions qui ont suivi Rockpolitick, les personnes présentes sur le plateau étaient invitées à donner leur avis sur la nouvelle émission. Les invités ont discuté de la satire, notamment la satirique politique, ses rapports avec le pouvoir, et se sont interrogés sur la possibilité de distinguer la bonne de la mauvaise satire. Les représentants du centre gauche, acquis à la cause de Rockpolitick, on souligné que la télévision publique souffrait d'un manque de pluralisme sous le gouvernement Berlusconi. En revanche, les représentants du centre droit ont critiqué Rockpolitick parce qu'elle proposait une satire à sens unique révélateur d'un message politique unilatéral. La question de fond est celle du pluralisme de l'information et de la liberté d'expression, face au conflit d'intérêt posé par Berlusconi et à l'anomalie de la situation italienne. Se pose aussi la question des règles à définir pour la prochaine campagne

\footnotetext{
1 Pour en savoir en plus : http://www.freedomhouse.org.
} 
électorale à la télévision, y compris le rôle de la satire. La discussion politique italienne en revient toujours à la télévision considérée à la fois comme un moyen d'expression pour les partis politiques, mais aussi comme un lieu de débat politique. Ce qui se joue dans ces débats est l'enjeu majeur de la liberté d'expression, de la démocratie italienne ${ }^{1}$. À ce sujet, les invités ont tenu à préciser qu'une émission de la télévision ne détermine pas le niveau de liberté d'un pays. Cependant, le professeur Manhaimer, expert en sondages, présentait aussi les résultats d'une enquête sur l'influence de Rockpoltick à propos du choix du parti politique aux prochaines élections. La confusion n'a pas disparu.

\section{Le court-circuit médiatique et la fin des genres télévisés}

Le cas Rockpolitick est le lieu privilégié pour observer l'état de la communication politique à la télévision italienne. Une émission de divertissement propose, dans son organisation et sa structuration, un espace important consacré à la politique, aborde des thèmes politiques et construit des événements d'impact politique.

Par ailleurs ce n'est pas seulement cette émission de spectacle qui est concernée, mais aussi l'émission d'information politique Porta a porta. L'émission traditionnellement consacrée à la politique italienne a eu la fonction de caisse de résonance de Rockpoltick. De surcroît, en étant focalisée sur le divertissement, elle est devenue elle-même spectacle, un spectacle fait par les politiques.

On comprend donc qu'il ne s'agit pas seulement de constater que des politiciens apparaissent maintenant dans des émissions de spectacle ou des talk-shows. En Italie, la phase médiatique qui voit les politiciens migrer vers le divertissement (et déserter les émissions d'information qui leur étaient traditionnellement consacrées) peut être considérée, à la lumière des faits récents, dépassée. Le nouveau phénomène est celui de l'émission politique même qui se transforme, change de genre et devient elle-même du spectacle, de façon toujours plus directe et consciente.

L'évolution de Porta a porta montre cette tendance. Le point fort de l'émission a toujours été de manier les caractéristiques des deux genres (information et divertissement). Au début, elle a représenté la nouveauté de montrer le côté humain des personnalités politiques en se positionnant dans un registre plus spectaculaire et personnalisé (cfr

1 Le ministre Buttiglione a souligné que l'Italie est évidemment un pays libre si « sur la chaîne publique on peut insulter le chef du gouvernement ». 
les exploits culinaires ou sportifs de Bossi, D’Alema et Amato). Elle a aussi élargi le débat politique aux interventions des hommes et (surtout) des femmes du spectacle. Ensuite, l'émission s'est intéressée à d'autres sujets sociétaux, notamment télévisuels (par exemple les reality shows). Enfin, nous avons vu que Porta a porta devient une sorte de double de son objet ; Rockpolitick.

La politique à la télévision ne se définit plus à travers ses espaces, les programmes consacrés à la politique et à l'information. Ce n'est ne pas une question de lieu télévisé. C'est la présence du politique qui fait d'une émission une émission politique. Le politique n'est plus un contenu, un thème, l'objet qui détermine le degré de politique d'un discours, mais le fait que le discours est prononcé par un homme ou une femme politique. Dans ce contexte, le genre perd son rôle et sa valeur.

Le jeu de miroirs entre Rockpolitick et Porta a porta génère un court-circuit médiatique qui confond les genres télévisuels. Le spectacle envahit la politique comme la politique elle-même envahit le spectacle. Les frontières sont brouillées. Les genres n'existent plus. La contamination n'a pas de limite. La deuxième émission de Porta a porta consacrée à Rockpolitick contient un moment emblématique : l'animateur, Bruno Vespa, montre des images tirées de Rockpolitick où l'on voit Celentano parler des politiciens qui, la semaine précédente, étaient invités à Porta a porta pour parler de Rockpolitick. Un jeu de poupées russes rendu possible justement par la disparition des contenus politiques et, par conséquence, des genres télévisés qui étaient structurés sur ce contenu.

Les raisons de ce phénomène médiatique sont complexes. Dans le registre politique, les interprétations sont variées et de natures différentes; nous pouvons néanmoins évoquer quelques éléments de réflexion au niveau médiatique. Actuellement, la télévision est toujours plus compétitive. La recherche de l'audience devient un principe structurant. Nous savons, grâce à des recherches menées sur ce sujet, qu'en général la politique intéresse les Italiens. La politique crée donc de l'audience, et encore plus, apparemment, quand les politiciens parlent du spectacle. En effet, Porta a porta a remporté le record d'audience dans une des émissions consacrées à Rockpolitick. La recherche de l'audimat peut donc expliquer l'abolition des genres puisqu'elle mène à l'autoréférence.

Nous avons déjà démontré que les émissions d'information sont devenues le lieu des politiciens, plus que celui de la politique, et que cette tendance laisse un vide dans la programmation télévisée. Nous 
avons aussi affirmé que ce vide semble pouvoir être comblé par les personnalités du spectacle qui construisent leurs shows autour des thèmes qui relevaient autrefois du territoire exclusif des politiciens. Si cette tendance venait à se confirmer, le risque est grand que la médiation du spectacle, ou la personnalisation du spectacle, devienne une nécessité afin de garantir l'efficacité de la parole politique à la télévision ${ }^{1}$. Le danger est donc, en dehors des périodes de campagne électorale, que les thèmes politiques disparaissent totalement.

1 La célèbre analyse de Walter Benjamin, dans «L'oeuvre d'art à l'ère de sa reproductibilité technique», sur l'esthétisation de la politique comme clé du fascisme, rappelle ce danger. 
